\title{
Should We Retire the Catalog?
}

\section{Dianne Cmor and Rory Litwin, Guest Columnists}

Dianne Cmor is Deputy University Librarian at Nanyang Technological University, Singapore. Rory Litwin is a former academic librarian and is now the owner of Library Juice Academy and Library Juice Press.

Correspondence concerning this column should be addressed to Karen Antell, Head of Outreach and Strategic Initiatives, and Molly Strothmann, Social \& Behavioral Sciences Librarian, University of Oklahoma Libraries, 401 W. Brooks St., Norman, OK 73019; email: kantell@ou.edu and mstrothmann@ou.edu.
As more libraries adopt web-scale discovery services, many now find themselves offering two options for searching their holdings: the new discovery tool and the traditional OPAC. Are both necessary? Or does a discovery system by itself provide an adequate search environment, making the catalog superfluous? In this installment of "Taking Issues," Dianne Cmor argues that investing in two search tools is an unwise use of limited resources. Rory Litwin counters that the direct control traditional OPACs offer is a necessity for librarians and other advanced researchers.-Editor

\section{CMOR}

With the overwhelming adoption of discovery systems by both libraries and library users, one has to ask-do we still need OPACs? I argue that they are not worth maintaining if we adequately integrate our records into discovery systems.

Like many librarians, I still believe there is an important place in research for sophisticated native databases with advanced, discipline-specific search features, such as Medline, SciFinder, Historical Abstracts, etc. But who among us has ever described our library catalogs in that way? We have long lamented the flaws and limitations of the OPAC modules of our library management systems. Although discovery systems are far from perfect (and I look forward to their improving over time), they do allow our users to find our owned and subscribed materials in a way that is easy and intuitive, including the items that could otherwise be found using the catalog. The library catalog does offer librarians and other expert searchers some options that can make the process of finding known items easier and more accurate (namely field searching and field browsing for title, author, and subject), but such features are not intuitive to our users. Moreover, the necessarily generic advanced search features of our library catalogs are not integral to sophisticated, comprehensive searching of our holdings (as in the databases I mention above) and do not offer the same added value that would make them worthwhile to sustain. Obviously, we still need back-end catalogs (or the equivalent) to feed our holdings into our discovery systems, but the user interface is no longer necessary.

\section{LITWIN}

I think whether OPACs are still necessary depends on the type of user and the nature of the research question. At a presentation by a representative of one of the major ILS vendors 


\section{TAKING ISSUES}

introducing their discovery system, I recall being told that the discovery system was the result of extensive user studies of undergraduates and was geared toward their needs. That is, the system was designed to help undergraduates find useful resources without a librarian's help. It was not designed for the needs of advanced researchers, who often have much more specific requirements than undergraduates searching for "something useful" for a research paper.

At my institution, we were encouraged to use the discovery tool when helping students. I noticed that when I wanted to help students find something based on my interpretation of their needs and my personal knowledge of the field they were researching, the discovery tool presented an obstacle. It was often harder to find the specific items that I wanted. So, while this tool is useful for discovery, especially for undergraduates, it is not as helpful for users who want to leverage the knowledge they already have of an area of study or of the collection. Therefore, I think we need to maintain-and, I hope, improve-some tool that is geared toward knowledgeable searchers. I think it is important to understand that discovery tools are designed to substitute for much of the work that knowledgeable librarians do in helping users. Sometimes they do that well, but it depends on what the user needs from the interface.

\section{CMOR}

I, too, have experienced the occasional frustration of not easily finding a specific item that I know we have in our collection-but this happens quite rarely, and usually as a result of poor mapping or insufficient or incorrect catalog data. When reported, these problems can usually be fixed. Instead of working to improve our catalogs, I would suggest that we insist that our discovery tools improve at known-item searching-for example, changing algorithms so that matching book titles are highly relevant and show in the first few hits. As you note, discovery tools are strong when it comes to easy and simple "discovery." They are also excellent tools for interdisciplinary topics, current topics, and unique topics because titles and subject headings are often not adequate to uncover these types of books. For these types of topics, discovery tools are highly useful because they provide enriched publisher metadata in central indexes and, for some items, full-text searching.

I agree that advanced researchers need advanced tools for comprehensive searching. I also agree that discovery tools cannot (currently) replace, for example, Medline for sophisticated, specialized searching. However, I do believe that they can adequately replace some databases with weaker search features, such as the MLA Bibliography (although its content is invaluable to literature majors such as myself, of course). In my opinion, library catalogs are in the same category. Although the "next generation" catalogs that came out just before discovery systems were certainly an improvement, I'm not convinced they are good enough to persuade me to pay for both.

\section{LITWIN}

I agree that traditional Boolean-based search tools work well only to the extent that the data structures are used consistently when their records are created, and poor cataloging reduces the power of traditional search tools more than it affects the newer discovery tools. This illustrates the fact that Boolean-based tools require knowledgeable users to work well, whereas discovery tools require less knowledge from their users to be effective. This is one of the points of appeal for novice searchers. The cost, however, is that they make it more difficult for advanced, knowledgeable users to take advantage of their expertise.

The knowledge that counts is not just knowing how to use Boolean operators and knowing what the different fields are-it's expertise about the research subject and the contents of the library collection. The advantage of the older "manual transmission" tools (to use an analogy that I like) is that they provide direct control of the search. If users cannot control what the search is doing, they must rely on the system to do some of the thinking for them. When the user's requirements fit the expectations that were used to program the tools, that's not a problem. The problem is when the smart system begins to impede the search attempts of expert users. As the discovery tools were not created with librarians in mind, they disregard the fact that what reference librarians have to offer is not just the mechanics of how old-style catalogs work, but knowledge of subject matter and of collections that we can use to help people in ways that discovery tools can't. Reference librarians and other expert users sometimes need the option to take control of their searches_-to "outsmart" the smart systems.

\section{CMOR}

So I think we both agree on a couple of points. First, search tools that are built on and use sophisticated taxonomies and structures should be retained for serious research. Second, librarians have subject and collection knowledge that adds great value to the work of researchers. But the OPAC is for the "public" primarily, not for librarians. Also, OPACs are broad in subject scope and do not take advantage of specialized search features (exploding of taxonomies, discipline-specific fields, etc.). So what exactly are the advantages of OPACs to users, both novice and more advanced searchers? And are those advantages (which I do believe exist) significant enough to justify expending the resources necessary to maintain the OPAC?

\section{LITWIN}

In planning for systems to search our holdings, the first question should be, "Who is the user?" We seem to disagree about that answer: I think that the OPAC is for reference librarians as well as for the "public." Different types of users with different requirements use the same research tools. Reference 
librarians are among those users, and so are people whom I would call advanced users, researchers who are searching for specific items (known or not) rather than simply looking for "something" that fulfills a broadly defined need. These include graduate students, professors in certain fields, and others doing serious bibliographic research over an extended period. That might imply that large research libraries have the greatest need to maintain the OPAC, but in my view, medium-size research libraries also serve a significant number of people with similar advanced research needs.

With regard to serving advanced users, I think the specific advantage of the OPAC is that it allows direct control over the searches. Researchers can use the structures of the database with clear Boolean terms, enabling them, for instance, to browse all items that have been assigned a specific subject heading, look at the most relevant ones to pick better subject headings, see all other titles by a specific author, follow the threads of coauthors, see titles in a series, identify technical keywords that they want to find in a title, and use resources that are external to the OPAC to learn about specific items to look up (known items, especially from bibliographies and references). Although it is possible to use most of these strategies in the newer discovery tools, they don't function as precisely because the basic search engine uses algorithmic term weighting instead of clear-cut Boolean inclusion/exclusion that gives the user precise control. Furthermore, some discovery tools don't even make some of these advanced features available.

\section{CMOR}

I agree that there is room for improvement in the features and functionality of discovery systems, but I'm not convinced that the majority of our researchers are still searching in the ways they once did. Reviewing the logs of catalog searches a couple of years ago, I rarely saw a precise search properly executed. More often, I saw "advanced" functions being used improperly, such as subject searches performed without using proper subject headings. Perhaps this is different now that most general searches are going through discovery tools and only the more advanced searchers go to the OPAC. Perhaps now we might see more properly constructed, precise searches in the logs because they are no longer being buried by all of those simple searches. If this were the case, I might have to reconsider my position, but I doubt that we would see this pattern.

I do find that most of my precise and specific needs can be met by discovery platforms, most of which allow for presearch field selection and post-search format limiting. When I cannot find what I am looking for, the problem often can be traced to incorrect or insufficient mapping. Following up on problems is time consuming, but often results in hundreds of records become more discoverable by fixing a generic mapping problem. Unfortunately there are times when library staff, upon failing to find something in the discovery system, quickly default back to the OPAC instead of reporting and correcting problems. This reinforces the superiority of the OPAC instead of improving the discovery system.

In a world of unlimited human and financial resources, I would agree that we should provide as many paths and options for our diverse users as possible. However, in a world of limited resources and changing areas for library services, we must weigh the impact and value of the work we do and the systems we maintain. If we continue to provide all the "useful" precision that is possible when the use of this type of precise searching is declining-how useful is it really? How much impact does the availability of such a tool have? To the minority of advanced searchers - quite a lot! And I would love to be able to take care of all the needs of all user groups no matter their size, but I don't think this is feasible. I think that, if we are going to put effort into advanced searching features, we should focus on the tool used most, so all users have to learn only one tool instead of two. And let's spare ourselves the time-consuming redundancy of maintaining two systems.

\section{LITWIN}

You make some very good points. I would acknowledge that at my institution, many of the frustrations we encountered with the discovery system could have been attributed to mapping problems. However, as it was explained to us by the head of technical services, the discovery system didn't allow for direct mapping of the MARC fields in the same way that the OPAC did. I am not an expert in the details of how these systems use MARC data, but if that were the case, and it was the source of the problem, it is an example of an area where our discovery system could have been improved. Also, if there are shortcomings of the current discovery systems that could be remedied more quickly if the vendors had more contact with frontline reference librarians (to whom they tend not to talk), then that would be another reason to argue that discovery systems aren't a problem in principle but should be seen in terms of their potential for improvement. So I acknowledge that point: discovery systems can potentially work better than they have worked for many of us.

That said, in debating this topic, I have mostly been thinking about discovery systems and traditional OPACs in terms of the principles behind them as I understand them: discovery tools provide help to the user by putting some complex programming into the interface, which connects the user to the data through a search engine that uses term weighting and other techniques. Much of this programming is aimed at doing some of the thinking for the user. That is in contrast to the comparatively simpler programming behind a traditional OPAC, which gives the user a direct mechanical connection between the interface and the data, returning records using Boolean matching. What I want to see preserved for users is that direct mechanical control of the search. The programming behind this approach is much simpler. If this kind of a search alternative could be built in to the discovery tool as an optional interface, I would have no problem giving up the 


\section{TAKING ISSUES}

traditional OPAC, and then I think the economic problem of maintaining two distinct software packages would be solved.

Regarding your argument that, given limited resources, we should concentrate on serving the majority of users, I admit to a somewhat elitist perspective: I think the more socially significant use of library resources generally results from the work of the advanced-search minority. I don't mean that we shouldn't support the needs of novice searchers. However, other users can be very important as well, even if there aren't as many of them. One way to think of this balance without the elitist-populist dichotomy is to think about the way university libraries serve the needs of undergraduates at the same time they serve the research needs of faculty. Although I would not say that the needs of researchers are always going to be best served by a Boolean search tool, these users are more likely to need the precision and known-item searching capabilities that are most easily accomplished through a direct, mechanical, controllable Boolean search (whether it is a complex or simple search expression).

Finally, we shouldn't overlook the needs of reference librarians, whom the designers of discovery tools do not view as necessary to serve the undergraduates who provide the profile for their designs. I believe that reference librarians, as search intermediaries applying their knowledge of their subjects and collections, contribute to student learning in ways that discovery tools do not. Reference librarians are able to help undergraduates identify resources that are appropriate for their work (or not) using knowledge that the students don't have yet and that the discovery tools do not have (even the smarter ones). Yes, it is certainly possible for librarians to use the discovery tools to provide this help, but my point is that developers of discovery tools leave librarians out of the equation in programming tools that prioritize making it easy for undergraduates to "find stuff." An alternative interface designed for reference librarians would optimally be a direct, mechanical interface like the traditional OPAC's. (And I am not exaggerating about the vendors leaving librarians out of the equation in designing these discovery tools. At a presentation I attended, the vendor presented their vision for the library reorganized around their products, and it included redefined roles for reference librarians that did not include helping undergraduates find materials.)

\section{CMOR}

I support all of the things that you have on your wish list for discovery systems: options for precision searching, an expanded view of who the "user" is to include serious researchers, and better communication between vendors and the librarians who are working directly with the full range of our users. I also agree with your excellent point that the needs of the masses do not outweigh the need for an interface that supports serious, socially significant research. As you have suggested, if discovery systems could provide an option for field-specific searching with precise inclusionexclusion options, that would help to meet the needs of all types of researchers, including librarians. I recognize that this is not currently the case, thus your strong argument to maintain the OPAC. However, if nothing changes, we will have to decide whether we can afford to maintain both systems and whether there is truly enough evidence to support that decision. Perhaps there is. Perhaps this may differ between institutions based on their user populations and user needs and behaviors. Perhaps a local OPAC is not the answer, but for serious researchers WorldCat or some other equivalent might be the future path.

\section{LITWIN}

Well, it does look like we agree on many points, including the fact that these questions are going to be answered differently at different institutions. I also think you're right to focus on the fact that it is basically an economic question: can libraries afford to maintain these systems? But you state an assumption for asking that question, which is "if nothing changes." I think we can be sure that these systems will continue to evolve, and if reference librarians lobby for improvements that support precise, controlled searching, we probably will see them. Ultimately, I think this is where we should focus our efforts, because the whole question we're debating may end up being moot if the vendors answer it for us simply by phasing out the traditional OPAC from their product lines. So, I think we've come to a good agreement, that the OPAC has search capabilities that we like and that we would like to see eventually incorporated in our discovery tools.

An interesting and related problem, I think, is that the economic challenges you cite regarding the OPAC could just as easily be raised about reference services in general: nice, but can we afford it? To a large extent, the case for OPAC-style search capabilities is also the case for reference librarians. But perhaps that is a topic for another debate. 